\title{
Data Acquisition and Alert System for Recirculation Aquaculture System (RAS) using Fog Computing
}

\author{
Siti Maryam Binti Zainol \\ Department of Electrical Engineering, Polytechnic Sultan Abdul Halim Mu'adzam Shah, \\ 06000 Jitra, Kedah, Malaysia.
}

\begin{abstract}
This research presents an improved and more effective approach for data acquisition of recirculation aquaculture system (RAS). The previous research, the system uses manual methods to take the important data from RAS and it wastes the time and also gets late response from the fish farmer if the data is not in the good condition. As a result, fog computing technology is applied to overcome all these problems and acts as advance data acquisition system to keep data safely by sharing the processed data in fog computing for every tanks and analyze the data to make an accurate control/decision in the real time. Besides, open source technology plus embedded system based will be integrated for this research because its benefits such as small size, low cost, light weight, portable, high efficiency and low power consumption. This research has achieved the objectives which are design a data collecting system for RAS, design a data processing system using fog computing and integrate, test and validate automatic data collection and processing strategy for recirculation aquaculture system (RAS). The data collecting system for RAS, RaspDAQ is developed by connecting Raspberry Pi 3 to temperature sensor (LM35DT) using analogue digital converter (ADC) MCP3002, water level sensor (HC-SR04), Rpi camera module, LEDs and buzzer. Software and program are built using Python and Apache server to run every functions of RaspDAQ. While third Raspberry Pi 3 is setup as data processing system, RaspFog using PHP, Apache and MySQL server. Both RaspDAQ and RaspFog are based on Raspbian operating system. After that, RaspDAQ1 and RaspDAQ2 are connected to RaspFog using WiFi technology to send sensors data in real time. The received data are stored and plotted using Highcharts.com graph. The data collecting system, RaspDAQ and data server and processor, RaspFog has been tested and validated. At the same time, users can see the graph output in the real time for temperature, water level sensor and real condition using Rpi camera module of RaspDAQ1 and RaspDAQ2 by browsing RaspFog website. From the observation, data has been transferred from RaspDAQ to RaspFog in a short duration which is less than 15 seconds. Consequently, the efficiency of data acquisition process has been improved from manual system to fog computing technology successfully.
\end{abstract}

Key words: $R A S$, fog computing, collecting system, data processing system

\section{INTRODUCTION}

Food and Agriculture Organization of the United Nations (FAO) [1] has reported that human consume more fish is instigated from farms than from wild capture, having reached almost parity in 2012 according to the latest global report. Because of that, the fishery and aquaculture sector have to face a critical defining moment when they need to move with the rapid technology changes, increasing demand, and rising feeding cost. Furthermore, the global projection of fish production is increasing from 2010 to 2030 by $24 \%$ which is 35.4 million tons as reported by Siwa Msangi and Miroslav Batka [2].

High density fish farming with Recirculation Aquaculture System (RAS) is said as key of technology which allows the world aquaculture community to supply the world per capita needs for aquatic species over the coming decades with environmentally and friendly manner. RAS also sustainable, infinitely expandable, environmentally compatible and has the ability to guarantee both safety and the quality of the fish produced throughout the year. On other hand, RAS offers the controlled environment, the permitting controlled product growth rates and predictable harvesting schedule. The heat and water can be conversed through the reused water after reconditioning by biological filtration [3].

Nevertheless, there are some parameters need to be monitored in RAS that can be divided into two aspects. The first aspect is the parameters of environment control such as temperature, Dissolve Oxygen (DO), $\mathrm{PH}$ and

Corresponding Author: Siti Maryam Binti Zainol, Department of Electrical Engineering, Polytechnic Sultan Abdul Halim Mu'adzam Shah, 06000 Jitra, Kedah, Malaysia. 
Ammonia (NH3) level to control the healthy environment for fish especially DO level which is the first limiting factor in intensive aquaculture system. It can immediately cause the fish fatality if DO is not at the required level. The second aspect is the parameters to be calculated to determine the fish growth and Feed Conversion Rate (FCR) such as the weight of fish, the weight of feed and the frequency of feeding. A real time and efficient monitoring system is really desired for some critical parameters which can improve the quality and determine future projection by using the stored data previously. Additionally, a very low latency is required to response the output device and to alert the stakeholder and aquaculture men if something unwanted condition happens such as temperature going higher. Secure and reliable system also high demanded due to high cost and profit business such as RAS.

Consequently, fog computing technology is suggested in this research to make RAS monitoring system is more efficient. Fog computing offers reduction of network traffic by providing a platform to filter and analyse the data generated by the devices which close to the edge and for local data views. As result, it will automatically reduce the traffic being sent to the cloud. Fog computing also suitable for Internet of Things (IoT) tasks and queries which most of the smart devices need to capture events only about a hundred meters from it, no need to access global data from cloud. Low latency requirement also provided by fog computing which critical parameter can be reflected with high speed real time response. Furthermore, it can reduce the scalability issue since fog computing aims the incoming data get closer to the data source itself and reduces the burden of that processing on the cloud and without concern of the increasing number of endpoints. [4].

\section{METHODOLOGY}

Research methodology is conducted in four subtopics including system overview and three phases as Figure 1 which start with data collecting, data processing, system integration and whole system testing.

\section{Phase 1}

Data collecting system is designed using Raspberry Pi (in this research, known as RaspDAQ (Raspberry Pi Data Acquisition Query)) connects with two sensors which are temperature sensor and water level sensor. In this research, two RaspDAQs are used to implement fog computing concept. This single board computer uses Raspbian (Linux) as operating system and the Python language to write the program.

\section{Phase 2}

This phase implements another Raspberry Pi (in this research, known as RaspFog) as sever in fog computing. Same with RaspDAQ, it uses same operating system, Raspbian. MySQL database is used for data record. Graphical User Interface (GUI) is built using PHP and HTML.

\section{Phase 3}

In final phase, the both of data collecting system, RaspDAQ1 and RaspDAQ2 send the data captured by two sensors for each RaspDAQ to RaspFog. At the same time, RaspFog stores the data in MySQL server and plots the graphs using the real time data from both RaspDAQ.

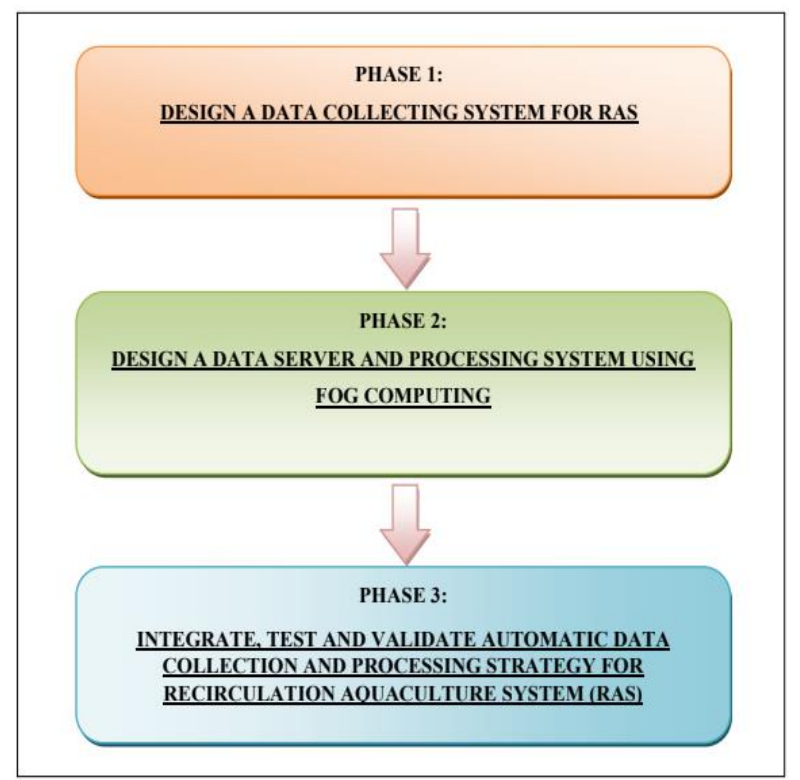

Figure 1 Three phases methodology for data acquisition and alert system for recirculation aquaculture system (RAS) using fog computing

\section{System Overview}

Automatic data acquisition for recirculation aquaculture system (RAS) using fog computing consists of data collectors which acts as client known as RaspDAQ and one server called as RaspFog as shown in Figure 2. In this research, both collectors and server use Raspberry Pi 3 as the platform. It is because it is very cheap, has friendly general purpose input output (GPIO) pin and use free operating system, Linux. In additional, it has the most important and special feature in this research which is $802.11 \mathrm{n}$ Wireless LAN.

RaspDAQ collects the temperature and water level data and display at graphical user interface (GUI) of RaspDAQ by real time. At the same time, the data and 
the camera input is sent to RaspFog using $\mathrm{WiFi}$ and store in MySQL server. RaspFog generates graphs for the temperature vs. time and water level vs. time. The GUI of server can be display by browsing the server website from any devices as they are connected to the same network.

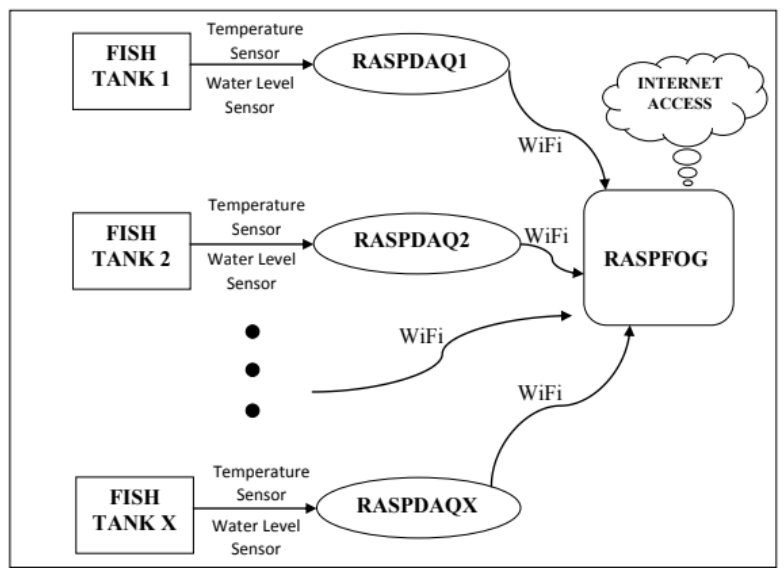

Figure 2 Overview of data acquisition and alert system for recirculation aquaculture system (RAS) using fog computing

\section{RESULT AND DISCUSSION}

Figure 3 shows two data collecting systems for RAS, RaspDAQ1 and RaspDAQ2 are setup successfully. Each RaspDAQ has temperature sensor connects to Raspberry $\mathrm{Pi}$ through analogue digital converter (MCP3002), camera module, LEDs and water level sensor (HC-SR04).

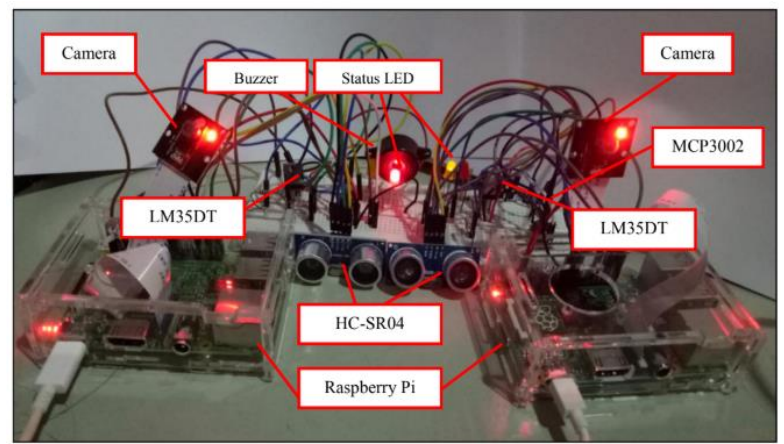

Figure 3 Data collecting system for RAS, RaspDAQ

Next, Figure 4 shows the graphical user interface (GUI) for RaspDAQ using Tkinter library as designed. It displays the system name and version, current date and time, current temperature, temperature average, minimum temperature, maximum temperature, current water level, water level average, minimum water level, maximum water level and system's up time. The temperature values are in unit ${ }^{\circ} \mathrm{C}$ and ${ }^{\circ} \mathrm{F}$. The system's up time start counting when the system is power on.

\begin{tabular}{|c|c|}
\hline \multicolumn{2}{|c|}{ RaspDAQ2 Ver 1.1} \\
\hline Date: & 2017-05-16 \\
\hline Time: & 1159 AM \\
\hline Current Temperature: & $35.45^{\circ} \mathrm{C} / 95.81^{\circ} \mathrm{F}$ \\
\hline Average Temperature: & $35.45^{\circ} \mathrm{C} / 95.81^{\circ} \mathrm{F}$ \\
\hline Minimum Temperature: & $35.45^{\circ} \mathrm{C} / 95.81^{\circ} \mathrm{F}$ \\
\hline Maximum Temperature: & $35.45^{\circ} \mathrm{C} / 95.81^{\circ} \mathrm{F}$ \\
\hline Current Water Level: & $80.86 \mathrm{~cm}$ \\
\hline Average Water Level: & $81.24 \mathrm{~cm}$ \\
\hline Minimum Water Level: & $81.1 \mathrm{~cm}$ \\
\hline Maximum Water Level: & $81.33 \mathrm{~cm}$ \\
\hline Up Time: & $00: 02: 98$ \\
\hline
\end{tabular}

Figure 4 Graphical user interface (GUI) for RaspDAQ

Figure 5 shows the sample of data capturing screenshot for RaspDAQ temperature test and validation. The capturing loops for every second to see the data consistency.

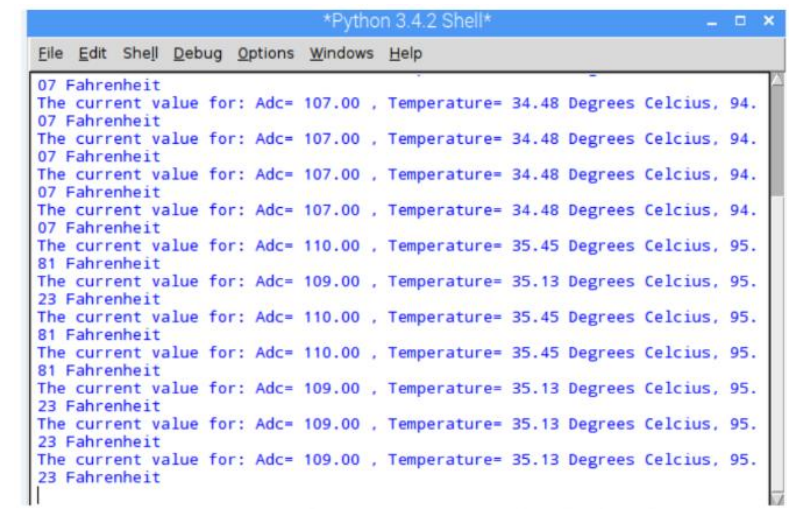

Figure 5 Data capturing for temperature test and validation of RaspDAQ

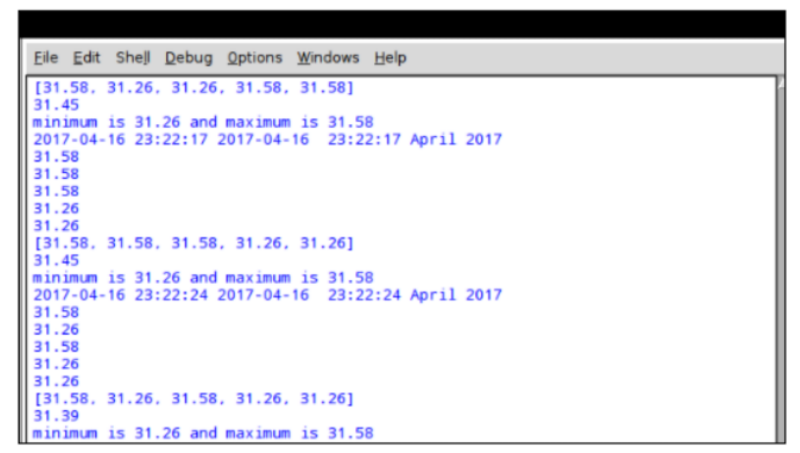

Figure 6 Data capturing for RaspDAQ average, minimum and maximum temperature. 
Figure 6 shows the data capturing screenshot for RaspDAQ average, minimum and maximum temperature. The capturing also loops for every second to see the data consistency. This program is also implemented to water level average, minimum and maximum.

Welcome page offers many access options which are displaying graph of RaspDAQ temperature sensor, water level sensor and RaspDAQ condition view / real time video as shown in Figure 7,8 and 9.

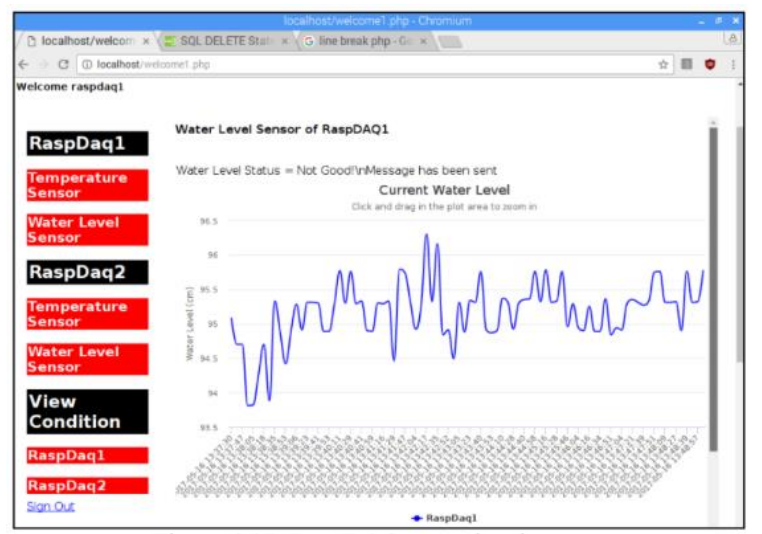

Figure 7 RaspDAQ water level sensor page

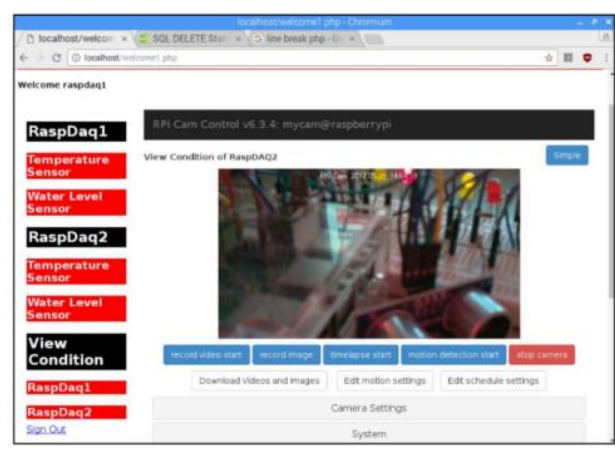

Figure 8 RaspDAQ condition view page

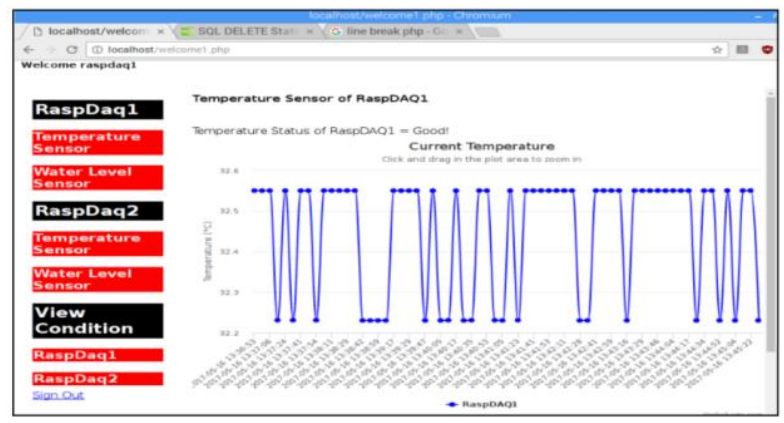

Figure 9 Current temperature against date, time
Figure 10 shows received email example while Figure 11 shows the received SMS example. The email can be sent to many recipients and SMS only can be sent to Twilio verified number.

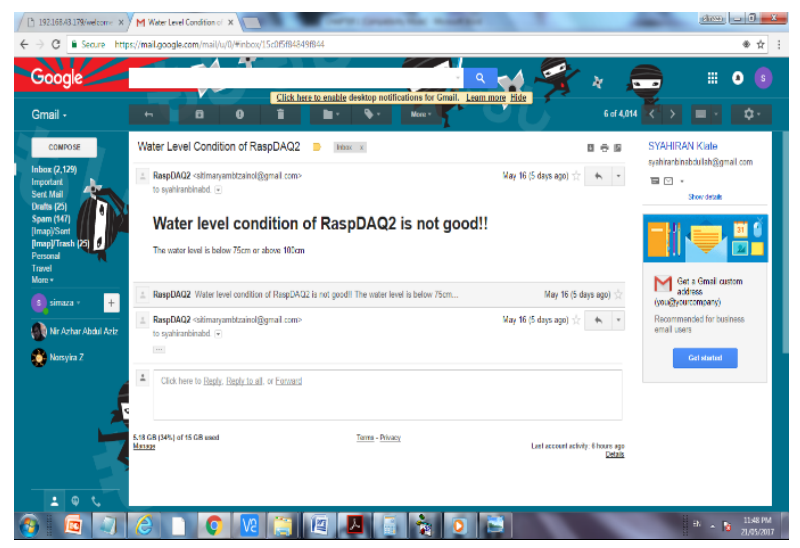

Figure 10 Received email from RaspFog system

\begin{tabular}{|c|c|c|}
\hline$<$ & 63001 & Details \\
\hline \multicolumn{3}{|c|}{ below $75 \mathrm{~cm}$ or above $100 \mathrm{~cm}$} \\
\hline \multicolumn{3}{|c|}{$\begin{array}{l}\text { RMO.00 Sent from your Twilio trial } \\
\text { account - Water level condition of } \\
\text { RaspDAQ1 is not good!!!t is } \\
\text { below } 75 \mathrm{~cm} \text { or above } 100 \mathrm{~cm}\end{array}$} \\
\hline & Tue $1605 / 201712: 49$ & \\
\hline & $\begin{array}{l}\text { M0.00 Sent from your Twilio trial } \\
\text { ccount - Water level condition of } \\
\text { aspDAQ1 is not good!llt is } \\
\text { elow } 75 \mathrm{~cm} \text { or above } 100 \mathrm{~cm}\end{array}$ & \\
\hline & $\begin{array}{l}\text { ent from your Twilio trial } \\
\text { ccount - Temperature condition } \\
\text { f RaspDAQ2 is not good!!lt is } \\
\text { elow } 25^{\circ} \mathrm{C} \text { or above } 33^{\circ} \mathrm{C}\end{array}$ & \\
\hline & $\begin{array}{l}\text { MO.00 Sent from your Twilio trial } \\
\text { ccount - Water level condition of } \\
\text { aspDAQ2 is not good"llt is } \\
\text { elow } 75 \mathrm{~cm} \text { or above } 100 \mathrm{~cm}\end{array}$ & \\
\hline & |rext message & $\operatorname{sen}$ \\
\hline
\end{tabular}

Figure 11 Received SMS from RaspFog system

Every phase of data acquisition and alert system for recirculation aquaculture system (RAS) using fog computing is tested and validated successfully. 


\section{CONCLUSION}

The data collecting system for RAS, RaspDAQ is developed by connecting Raspberry Pi 3 to temperature sensor (LM35DT) using analogue digital converter (ADC) MCP3002, water level sensor (HC-SR04), Rpi camera module, LEDs and buzzer. Software and program are built using Python and Apache server to run every functions of RaspDAQ. While third Raspberry Pi 3 is setup as data processing system, RaspFog using PHP, Apache and MySQL server. Both RaspDAQ and RaspFog are based on Raspbian operating system. After that, RaspDAQ1 and RaspDAQ2 are connected to RaspFog using WiFi technology to send sensors data in real time. The received data are stored and plotted using Highcharts.com graph. It is very interactive and beautiful graph interface. The data are also tested to alert the users about the limited values of the sensors by email and SMS.

Besides, data has been transferred from RaspDAQ to RaspFog in a short duration which is less than 15 seconds. Finally, RaspDAQ and RaspFog are tested and validated to confirm that data acquisition and alert system for recirculation aquaculture system (RAS) using fog computing is successful developed and implemented. The efficiency of data acquisition process has been improved from manual to fog computing technology successfully.

\section{REFERENCES}

[1] Food and Agriculture Organization of the United Nations. (2014). The State of World Fisheries and Aquaculture, 2014: Opportunities and Challenges. [2] Siwa Msangi and Miroslav Batka, (2015). Chapter 8, 2014 - 2015 Global Food Policy Report., https://www.ifpri.org/sites/default/files/gfpr/2015/featu re 3087.html, retrieved on 1 April 2016

[3] Tidwell, J. H. (2012). World Aquaculture Society Book series: Aquaculture Production Systems (1).

Hoboken, US: Wiley-Blackwell, http://www.ebrary.com, retrieved on 1 April 2016 [4] Amir Vahid Dastjerdi, Harshit Gupta, Rodrigo N. Calheiros, Soumya K. Ghosh, \& Rajkumar Buyya. (2016). Fog Computing: Principles, Architectures, and Applications. arXiv:1601.02752v2, 1-26. Retrieved March 25, 2016 from http://arxiv.org/abs/1601.02752v2 